\title{
Euphorbia hirta Linn (Euphorbiaceae), une espèce végétale prometteuse pour combattre les dysenteries bacillaires: expérimentations in vitro en Côte d'Ivoire
}

\author{
Allali Eugène KOFFI ${ }^{1 *}$, P. Abba OBOUAYEBA ${ }^{1}$, S. T. Djako AKRE ${ }^{1}$, \\ Loukou Manassé KOUADIO ${ }^{1}$, A. SAKIRIGUI ${ }^{2,3}$, Adolphe Mocket EHOUMAN ${ }^{4}$ et \\ A. B. Jacques Auguste ACKAH ${ }^{1}$ \\ ${ }^{1}$ UFR Agroforesterie, Université Jean Lorougnon GUEDE, BP 150 Daloa, Côte d'Ivoire. \\ ${ }^{2}$ Laboratoire de Pharmacognosie et des Huiles Essentielles, Facultés des Sciences et Techniques, Université \\ d'Abomey Calavi, 01 BP: 918 ISBA Cotonou, Bénin. \\ ${ }^{3}$ Laboratoire de Recherche sur les Plantes Aromatiques, Alimentaires et Médicinales à la Faculté des Sciences \\ et Techniques de l'Université Nationale des Sciences, Technologies, Ingénierie et Mathématiques (UNSTIM), \\ République du Bénin. \\ ${ }^{4}$ Olopam Pharma and Research \& Development, Côte d'Ivoire. \\ *Auteur de correspondance ; E-mail: koffiallali@yahoo.fr; Tel.: (+225)0749614728 / 0566679105
}

Received: 24-01-2021 $\quad$ Accepted: 31-07-2021 $\quad$ Published: 31-08-2021

\section{RESUME}

Les shigelloses (dysenterie bacillaire) persistent actuellement sous forme endémique dans les pays tropicaux et sont accompagnées d'échecs thérapeutiques à cause des souches de Shigelles (agents pathogènes) résistantes aux antibiotiques, constituant ainsi une priorité de santé publique. En Côte d'Ivoire, dans les recherches d'alternatives thérapeutiques pour éradiquer ce fléau, les activités antishigella in vitro, d'extraits d'Euphorbia hirta, plante utilisée en médecine traditionnelle pour guérir les diarrhées sanglantes ont été comparées. Un extrait total aqueux (ETaq) a été obtenu par macération de la poudre végétale dans de l'eau distillée et un extrait hydroéthanolique (EETH70\%) a aussi été obtenu par macération de la poudre végétale dans l'éthanol70\%. Les tests bactériologiques ont été effectués sur les souches cliniques de Shigella sp (EEQ ; 1055) pour déterminer les Concentration Minimale Inhibitrice (CMI) et Concentration Minimale Bactéricide (CMB). Aussi, les rapports d'efficacité des différents extraits sur la base des $\mathrm{CMB}$, ont été recherchés. Ces travaux ont montré que les valeurs des CMIs varient de 6,25 à $12,5 \mathrm{mg} / \mathrm{mL}$ tandis que celles des CMBs sont de $25 \mathrm{mg} / \mathrm{mL}$ pour l'E.ETH70\% (respectivement contre les deux souches :1055 et EEQ de Shigella sp.). Les CMIs et CMBs prises dans ce même ordre sont respectivement de $50 \mathrm{mg} / \mathrm{mL}$ pour l'E.Taq. (contre les deux souches) et de 100 à $200 \mathrm{mg} / \mathrm{mL}$ respectivement contre les souches EEQ et 1055. Toutefois, l'E.ETH70\% est quatre à huit fois plus actif que l'E.Taq. respectivement pour les inhibitions des souches cliniques EEQ et 1055 (par référence au rapport d'efficacité sur la base des CMBs, soit $\left.\mathrm{CMB}_{\mathrm{E} . \mathrm{Taq}}\right) /(\mathrm{CMB}$ ЕETH70\%, montrant ainsi que ces souches testées sont plus sensibles à l'E.ETH70\%. Ces travaux constituent une assise scientifique à l'usage traditionnel de cette plante, dans le traitement des diarrhées sanglantes.

(C) 2021 International Formulae Group. All rights reserved.

Mots clés : Activités antibactériennes, Souches cliniques, anti-Shigella. Shigellose. 


\title{
Euphorbia hirta Linn (Euphorbiaceae), a promising plant species for controlling bacillary dysentery: in vitro experiments in Côte d'Ivoire
}

\begin{abstract}
Shigellosis persist in endemic form in tropical countries. The development of antibiotic resistance in shigella has resulted in increased failure rates of treatment of shigellosis caused by those bacteria, as drugs to which they were previously susceptible no longer works. Shigellosis constitute thus, a public health issue. In Côte d'Ivoire, alternative therapeutic for eradication of this social plague, have lead us to comparative studies of in vitro antishigella activities of extracts from Euphorbia hirta, plant used by traditional healers to cure bloody diarrhea. Powder of dried leaves of this plant are extracted by maceration in distilled water to obtain a total aqueous extract: (ETaq). The ethanolic extract (E.ETH70\%) is obtained from vegetable powder as the same way as precedent in $70 \%$ ethanolic solution. Bacteriological tests were carried out on the clinical strains of shigella sp.(EEQ; 1055), to determine Minimum Inhibitory Concentration (MIC) and Minimum Bactericidal Concentration (MBC), supported by a searching of the ratio of effectiveness of these extracts on the basis of MBC.The results show that both extracts tested have in vitro antibacterial bactericidal efficiency on both Shigella sp.strains. However, the 70\% ethanolic extract (E.ETH70\%) which is four to eight times as effective as the total aqueous extract is more active against strains of Shigella sp tested, with the MICs varying from 6.25 to 12.5 $\mathrm{mg} / \mathrm{mL}$ while those of the MBCs are $25 \mathrm{mg} / \mathrm{mL}$ (respectively against the two Shigella sp. strains: 1055 and EEQ) vs MICs and CMBs taken in this same order are respectively $50 \mathrm{mg} / \mathrm{mL}$ for E.Taq. (against both strains) and 100 to $200 \mathrm{mg} / \mathrm{mL}$ respectively against EEQ and 1055 strains. This work scientifically validate the traditional use of Euphorbia hirta as a natural antidiarrheal substance in the treatment of bloody diarrhea diseases (shigellosis).
\end{abstract}

(C) 2021 International Formulae Group. All rights reserved.

Keywords: Antibacterial activity, Clinical strains, anti-Shigella, Shigellosis.

\section{INTRODUCTION}

Depuis toujours, les plantes ont été utilisées dans la médecine traditionnelle pour prévenir ou traiter plusieurs pathologies. Durant ces deux dernières décennies, la recherche en phytothérapie est devenue une des plus grandes préoccupations scientifiques (Anne-Sophie, 2018) et l'enquête ethnobotanique s'est avérée l'une des approches la plus fiable pour la découverte de nouveaux médicaments (Bisht et al., 2010 ; Yolidje et al., 2020).

En effet, les plantes médicinales contiennent des molécules bioactives qui présentent des intérêts multiples mis à profit dans différents domaines. Parmi ces composés, figurent les métabolites secondaires qui se sont surtout illustrés en thérapeutique (Koudoro et al., 2019 ; Sekandi et al., 2020).

Malgré ce savoir-faire traditionnel et la médecine moderne, la diarrhée, deuxième cause de mortalité chez les enfants de moins de cinq ans, tue 525000 enfants chaque année (Aubry and Gaüzère, 2019). Ces maladies diarrhéiques constituent encore aujourd'hui, un problème majeur de santé publique dans les pays en développement. Les agents infectieux responsables de la diarrhée sont généralement propagés par la voie oro-fécale, surtout par l'ingestion d'eau ou d'aliments contaminés par les selles ou par un contact direct avec des selles infectées. En effet, 2,6 milliards de personnes (en 2009), soit la moitié du monde en développement, n'ont pas accès à une latrine et 1,1 milliard de personnes n'ont aucun accès à une source d'eau salubre. La conséquence directe est que 1,6 million de personnes meurent chaque année de maladies diarrhéiques (Aubry and Gaüzère, 2011).

Cependant, parmi les infections bactériennes se trouvent les gastroentérites qui font partie des pathologies infectieuses les plus graves chez l'Homme. Les espèces bactériennes responsables des gastro-entérites dont celles du genre Shigella nécessitent 
généralement un traitement antibiotique. La plupart des pays où la shigellose est endémique et/ou épidémique sont également et fortement touchés par le VIH/sida (Katarzyna et al., 2019). Les traitements classiques jusqu'alors recommandés sont soit une aminopénicilline soit le cotrimoxazole. Or, depuis plusieurs années, des souches de Shigella résistantes aux aminopénicillines et au cotrimoxazole ont émergé et sont devenues prédominantes (Robert et al., 2004 ; Naeemah et al., 2020).

La shigellose est ainsi un problème mondial de santé humaine et une cause majeure de diarrhée causant environ 700000 décès par an dans le monde. La plupart des décès concernent des enfants de moins de 5 ans (Tai et al., 2016).

Malgré l'existence des nombreux antibiotiques, le traitement de la shigellose heurte le problème de la multirésistance croissante aux antimicrobiens entraînant fréquemment des échecs thérapeutiques et se solde par des complications, voire la mort (OMS, 2004 ; Okou and Yapo, 2018).

Face à ces nombreux échecs thérapeutiques, la recherche des nouvelles séries de médicaments est devenue plus qu'une nécessité (Lehmann, 2014 ; Ouattara et al., 2020).

Le patrimoine floristique africain s'avère très riche en plantes médicinales dont l'efficacité est déjà reconnue. Et parmi ces plantes médicinales, figure Euphorbia hirta, utilisée en médecine traditionnelle pour traiter les diarrhées infectieuses ou non (Adjanohoun and Aké-Assi, 1979). Cette espèce végétale d'aussi grande importance pour la santé des populations mérite d'être étudiée scientifiquement pour sa meilleure utilisation.

Ainsi, la présente étude a été menée dans le but d'évaluer l'activité des extraits aqueux et hydroéthanolique de cette plante sur la croissance in vitro de deux souches cliniques de Shigella sp. impliquées dans des diarrhées sanglantes et de comparer leur activité pour un usage rationnel.

\section{MATERIEL ET METHODES Matériel \\ Matériel végétal}

Le matériel végétal est constitué de la plante entière d'Euphorbia hirta, plante herbacée. Cette plante a été sélectionnée et collectée dans son habitat naturel, aux alentours de l'Université Jean Lorougnon Guédé de Daloa (une ville de la région du Haut Sassandra qui est située entre $6^{\circ} 51^{\prime}$ et $7^{\circ} 24^{\prime}$ latitude Nord - $6^{\circ} 59^{\prime}$ et $7^{\circ} 10^{\prime}$ longitude Ouest) durant le mois de novembre à décembre 2018.

\section{Souches bactériennes}

Le support microbien est constitué de souches bactériennes pathogènes de Shigella sp. (EEQ-1055), fournies par le laboratoire d'analyses médicales du Centre Hospitalier Régional de Daloa (CHR). Ces souches ont été isolées à partir des selles de malades souffrant de dysenterie bactérienne.

Des géloses et bouillons Mueller Hinton ${ }^{\circledR} \quad\left(\right.$ Bio $\operatorname{Rad}^{\circledR}$, Marnes-la-Coquette, France) ont servi soit, à des tests bactériologiques, soit à des cultures. Les géloses Salmonella-Shigella ${ }^{\circledR} \quad$ (SS) et Hecktoen ${ }^{\circledR}$ ont servi à des tests de sélection des souches bactériennes.

$\mathrm{Du}$ matériel de laboratoire de microbiologie et appareillage ainsi que des solvants ont été utilisés.

\section{Méthodes \\ Préparation des extraits végétaux}

Les plantes entières d'Euphorbia hirta récoltées, ont été soigneusement lavées à l'eau, découpées en morceaux et séchées à la température ambiante au laboratoire d'agro valorisation de l'UFR Agroforesterie de l'Université Jean Lorougnon GUEDE, pendant 10 jours (à 1'abri du soleil). Celles-ci ont été ensuite pulvérisées finement à l'aide d'une broyeuse mécanique de type Retsch SK ${ }^{\circledR} 100$, afin d'obtenir une poudre végétale.

Les extraits ont été préparés selon la méthode de Miezan et al. (2017), (légèrement modifiée). Pour ce faire, cent grammes de poudre végétale sont extraits par macération dans un litre d'eau distillée sous agitation magnétique pendant vingt-quatre heures sur un agitateur de type RTC B, IKA ${ }^{\circledR}$ (Staufen ; Germany). L'homogénat obtenu a été filtré successivement deux fois sur un tissu blanc, deux fois sur du coton hydrophile puis deux fois sur papier filtre Whatman ${ }^{\circledR}$ (diamètre = $150 \mathrm{~mm}$, grade 0859, Schleicher \& Schüll, Selecta, Dassel, Germany). Le filtrat obtenu a 
été déshydraté à l'aide d'une étuve de type $\mathrm{PROLABO}^{\circledR}$ à la température de $60^{\circ} \mathrm{C}$ pendant 7 jours. La poudre obtenue est l'extrait total aqueux (E.Taq.). Il a été récupéré, stocké dans un bocal en verre enveloppé par du papier aluminium puis conservé au réfrigérateur à 4 ${ }^{\circ} \mathrm{C}$ pour les tests bactériologiques.

La préparation de l'extrait éthanolique $70 \%$ (E.ETH70\%) a été faite dans les mêmes conditions que celle de l'E.Taq. Mais, le solvant d'extraction « eau » a été remplacé par une solution éthanol absolu-eau; (70:30, $\mathrm{V} / \mathrm{V})$.

Pour chaque extrait obtenu, le rendement a été déterminé selon la formule cidessous :

\section{$R(\%)=M_{1} \times 100 / M_{0}$}

où $\mathrm{R}$ est le Rendement de l'extrait exprimé, en pourcentage (\%); $\mathrm{M}_{1}$, la Masse de l'extrait (g) et $\mathrm{M}_{0}$ exprime la Masse de poudre végétale $(\mathrm{g})$.

\section{Préparation et numération de l'inoculum}

A partir d'une culture stock, des souches de Shigella sp. codifiées (EEQ et 1055) ont été repiquées séparément par la méthode des stries sur des boîtes gélosées de Mueller Hinton (M.H), puis incubées pendant 18 à 24 heures.

L'inoculum a été préparé à partir d'une colonie jeune de 18 à 24 heures parfaitement isolée et émulsionnée dans $10 \mathrm{~mL}$ de bouillon M.H.(BMH). Après homogénéisation, puis incubation pendant 5 heures à une température de $37{ }^{\circ} \mathrm{C}$, l'on obtient une pré-culture. Un (1) $\mathrm{mL}$ de cette pré-culture a été collecté pour chaque souche bactérienne et ajouté à $10 \mathrm{~mL}$ de BMH stérile. Cette suspension bactérienne développée a été évaluée à environ $10^{6}$ UFC/mL constituant ainsi l'inoculum pur (Miezan et al., 2017).

Détermination de la Concentration Minimale Inhibitrice (CMI) des différents extraits d'Euphorbia hirta

Elle se définit comme étant la plus petite concentration d'extrait de plante pour laquelle il n'y a pas de croissance visible à l'œil nu (Okou and Yapo, 2018).

Au premier jour pour chaque essai, huit (8) tubes à essai ont été utilisés pour la série test et huit (8) tubes pour la série de référence. Pour la série test, les sept (7) premiers tubes ont été numérotés de 1 à 7 et le dernier tube codifié
TC,a servi de tube témoin de croissance du germe bactérien. Pour la réalisation de ce test, il a été introduit dans les tubes numérotés de 1 à 6 et le tube TC, $1 \mathrm{~mL}$ de BMH. Ensuite, il a été transféré également dans les tubes 7 et 6, 1 $\mathrm{mL}$ d'extrait d'Euphorbia hirta, à raison de $800 \mathrm{mg} / \mathrm{mL}$ pour l'E.Taq ou $200 \mathrm{mg} / \mathrm{mL}$ pour l'E.ETH70\%, puis après homogénéisation, 1 $\mathrm{mL}$ du mélange a été reporté de tube en tube, de 6 à 1 (dont $1 \mathrm{~mL}$ du mélange est rejeté), à l'aide de la micropipette à embout stérile (PRO Accumex $\left.{ }^{\circledR}\right)$, selon une suite géométrique de raison $1 / 2$ (méthode de double dilution). Les concentrations intermédiaires finales obtenues varient de $800 \mathrm{mg} / \mathrm{mL}$ (tube 7) à $12,5 \mathrm{mg} / \mathrm{mL}$ (tube 1) pour l'E.Taq et de $200 \mathrm{mg} / \mathrm{mL}$ (tube 7) à $3,125 \mathrm{mg} / \mathrm{mL}$ (tube 1), pour l' l'E.ETH70\%.

$1 \mathrm{~mL}$ d'inoculum pur a été introduit dans chaque tube (de 1 à 7 et TC). Les concentrations finales varient ainsi de 400 $\mathrm{mg} / \mathrm{mL}$ à $6,25 \mathrm{mg} / \mathrm{mL}$ pour l'E.Taq et de 100 $\mathrm{mg} / \mathrm{mL}$ à $1,5625 \mathrm{mg} / \mathrm{mL}$ pour l'E.ETH70\%, avec un volume de $2 \mathrm{~mL}$ dans chaque tube.

Pour la réalisation de la série de référence, l'opération précédente a été suivie. Cependant en lieu et place de l'inoculum pur, il a été utilisé de l'eau distillée stérile. Ainsi, à la place du tube de contrôle de croissance TC de la série test, il y a un tube témoin de contrôle de stérilité (TS). L'ensemble des tubes de la série test et de la série de référence a été incubé pendant $18 \mathrm{~h}$ à $24 \mathrm{~h}$ à $37{ }^{\circ} \mathrm{C}$ et la $\mathrm{CMI}$ des différents extraits pour chaque souche a été déterminée (au deuxième jour) par comparaison tube par tube, concentration par concentration entre les tubes de la série de référence et ceux de la série test. Cette concentration a été déterminée à l'œil nu, à la lumière du jour, à la recherche d'une absence de turbidité ou de croissance visible (Okou and Yapo, 2018).

Ce même jour, une ou des boîte(s) témoin(s) (boîte A) est obtenue par numération de l'inoculum à partir des dilutions (d) de l'inoculum (TC, le tube témoin de croissance); ces dilutions sont exprimées par $\mathrm{d}$ : soit $\mathrm{d}=$ $1 / 10,1 / 100,1 / 1000$ et $1 / 10000$. La dernière dilution $\left(1 / 10000\right.$, soit $\left.10^{-4}\right)$ correspond à $0,01 \%$ de survivants, tandis que l'inoculum pur correspond à $100 \%$ de survivants, soit $(\mathrm{d}=$ $10^{0}$ ). Les différentes dilutions et TC sont 
ensemencés sur de la gélose M.H, suivant des traits de $5 \mathrm{~cm}$, réalisés au revers des boîtes de Pétri ${ }^{\circledR}$. Les boîtes sont incubées à $37{ }^{\circ} \mathrm{C}$ pendant 18 à 24 heures, puis conservées à $4{ }^{\circ} \mathrm{C}$ jusqu'à la fin des essais.

Détermination de la Concentration Minimale Bactéricide (CMB) des différents extraits d'Euphorbia hirta

La Concentration Minimale Bactéricide (CMB) est considérée comme la plus petite concentration d'extrait de plante pour laquelle, il y a $0,01 \%$ de Shigella sp. survivants, comparativement à la numération de l'inoculum de départ (Okou and Yapo, 2018).

Après la détermination de la CMI le deuxième jour, les contenus des tubes, ne présentant pas de trouble visible à l'œil nu et le témoin de croissance (TC) sont ensemencés de façon identique à celle des boîtes témoins et incubées à $37{ }^{\circ} \mathrm{C}$ pendant 18 à 24 heures : ce sont les boîtes essais. La CMB se lit au troisième jour, par comparaison du nombre de survivants des Shigelles de la boîte ou des boîtes essai(s), à ceux des boîtes témoins. En effet, Après $24 \mathrm{~h}$ d'incubation à l'étuve à $37^{\circ} \mathrm{C}$, le nombre de colonies sur les stries a été comparé à celui de la boîte de numération de l'inoculum (Boîte A ou boîtes témoin). Ainsi, le premier tube expérimental ou boîtes essai dont le nombre de colonies présentes sur sa strie est inférieur ou égal à celui de la dilution 1/10000 (du tube témoin de croissance (TC), correspond à la CMB.

Le Rapport CMB/CMI permet de démontrer l'efficacité d'un médicament. Selon Marmonier (1990) et Sirot (1990), lorsque ce rapport est inférieur ou égal à 4 , la drogue testée est qualifiée de bactéricide, mais s'il est supérieur à 4, la substance testée est bactériostatique en fonction de la souche bactérienne.

\section{RESULTATS}

\section{Rendement des extractions}

L'E.Taq. et l'E.ETH70\% ont été préparés à partir de cent (100) grammes de poudre végétale. Les masses moyennes obtenues, $\mathrm{M}_{1}=22,54 \mathrm{~g}$ correspondent à un rendement moyen $R_{1}=22,54 \%$ pour l'E.Taq. et $\mathrm{M}_{2}=12,21 \mathrm{~g}$, avec un rendement moyen $\mathrm{R}_{2}$ $=12,21 \%$ pour l'E.ETH70\%.

\section{Aspect des cultures bactériennes après repiquage sur milieu solide pour la détermination des paramètres antibactériens (CMI, CMB)}

Les Figure 1 et Figure 2 ci-dessous présentent les résultats de l'action de l'E.Taq sur la croissance in vitro des souches de Shigelles testées. Sur ces figures, les cultures obtenues sur les différentes stries au contact des concentrations de plus en plus élevées de cet extrait, ont des croissances qui se présentent généralement sous des formes de nappes de colonies denses selon la concentration de l'extrait utilisée. A la concentration de 200 $\mathrm{mg} / \mathrm{mL}$ de cet extrait, il y a absence totale de colonie de la souche de Shigella sp.1055 (Figure 1), tandis que pour la souche de Shigella sp. EEQ, il a été observé 4 colonies à la concentration $100 \mathrm{mg} / \mathrm{mL}$ (Figure 2). 200 $\mathrm{mg} / \mathrm{mL}$ et $100 \mathrm{mg} / \mathrm{mL}$ sont donc les CMBs (Figure 1 ; Figure 2).

Les Figure 3 et Figure 4 ci-dessous présentent les résultats de l'action de l'E.ETH70\% sur la croissance in vitro de souches de Shigelles. Les cultures obtenues sur les différentes stries au contact des concentrations de plus en plus élevées de cet extrait ont des croissances qui se présentent généralement, sous des formes de nappes de colonies denses ou non selon la concentration de l'extrait utilisé. A la concentration de 25 $\mathrm{mg} / \mathrm{mL}$ de cet extrait, il y a absence totale de colonie de la souche de Shigella sp.1055 (Figure 3); de même pour la souche Shigella sp.EEQ, l'absence totale de colonie s'observe à la concentration $25 \mathrm{mg} / \mathrm{mL}$ qui est donc la CMB (Figure 3 ; Figure 4). Aussi, le tableau 1 récapitule tous les paramètres antibactériens et les rapports $\mathrm{CMB} / \mathrm{CMI}$, CMBETaq/CMB E.ETH70\%. 


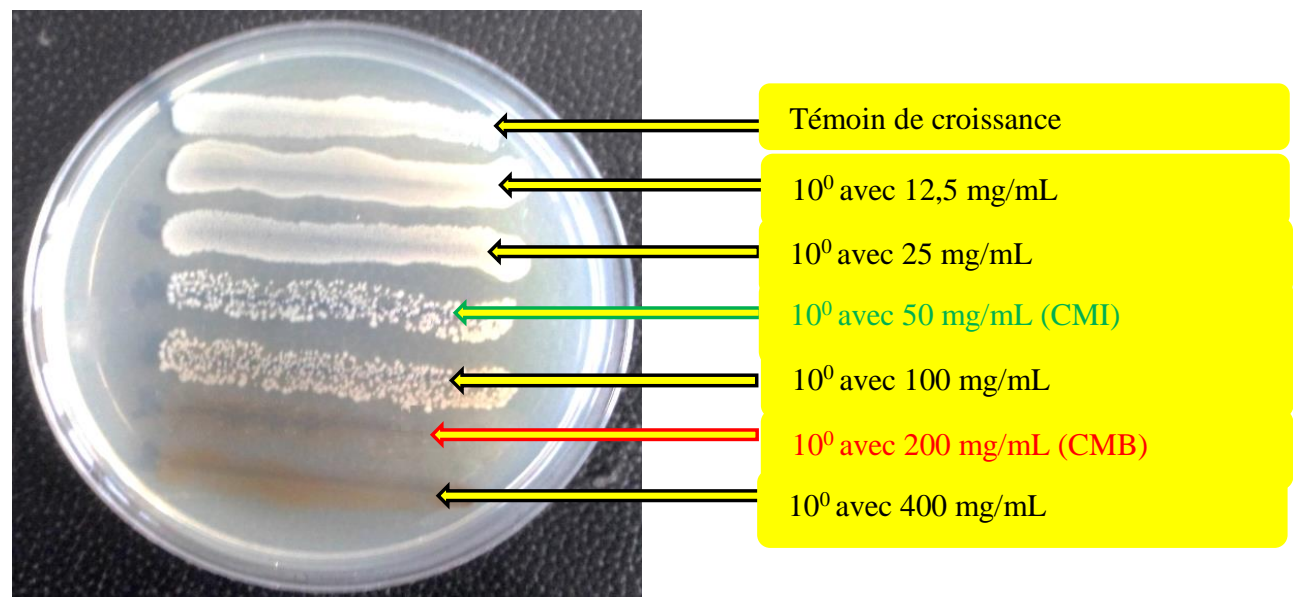

Figure 1: Action de l'extrait aqueux sur la croissance in vitro de la souche de Shigella sp.1055.

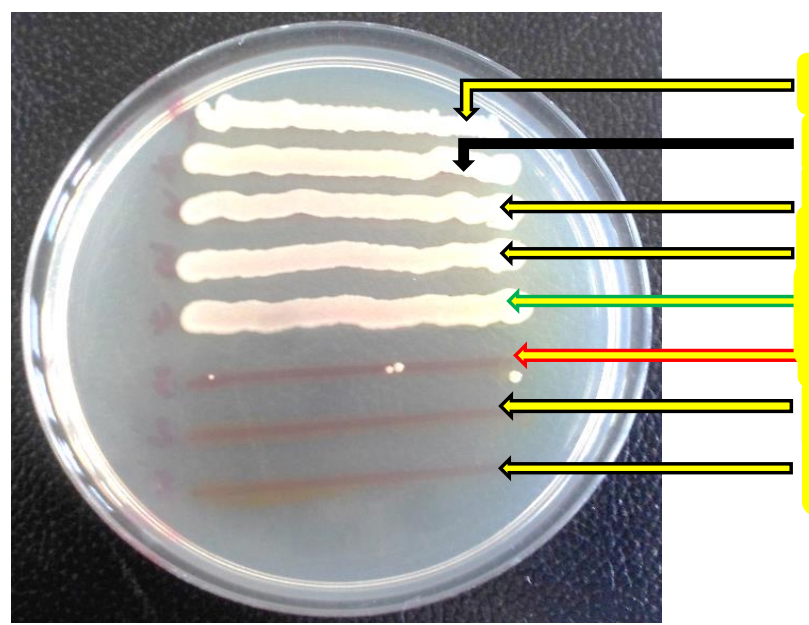

Témoin de croissance

$10^{0}$ avec $6.25 \mathrm{mg} / \mathrm{mL}$

$10^{0}$ avec $12.5 \mathrm{mg} / \mathrm{mL}$

$10^{0}$ avec $25 \mathrm{mg} / \mathrm{mL}$

$10^{0}$ avec $50 \mathrm{mg} / \mathrm{mL}(\mathrm{CMI})$

$10^{0}$ avec $100 \mathrm{mg} / \mathrm{mL}(\mathrm{CMB})$

$10^{0}$ avec $200 \mathrm{mg} / \mathrm{mL}$

$10^{0}$ avec $400 \mathrm{mg} / \mathrm{mL}$

Figure 2: Action de l'extrait aqueux sur la croissance in vitro des souches de Shigella sp EEQ.

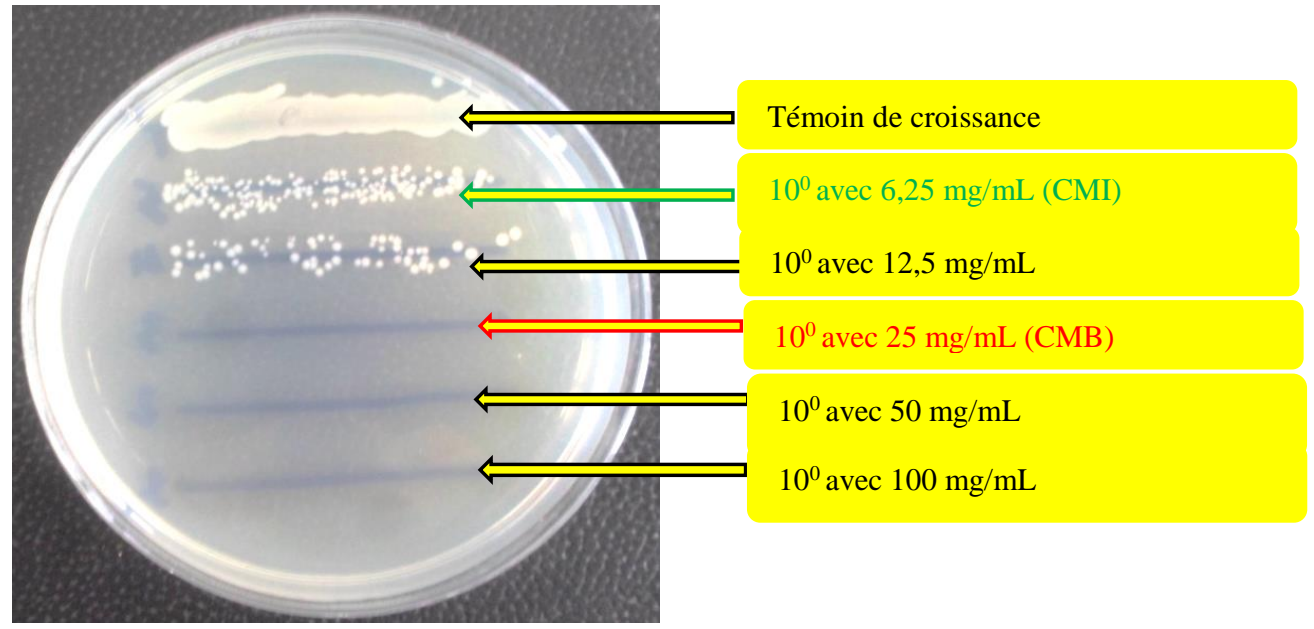

Figure 3: Action de l'extrait hydroéthanolique sur la croissance in vitro des souches de Shigella sp. 1055 . 


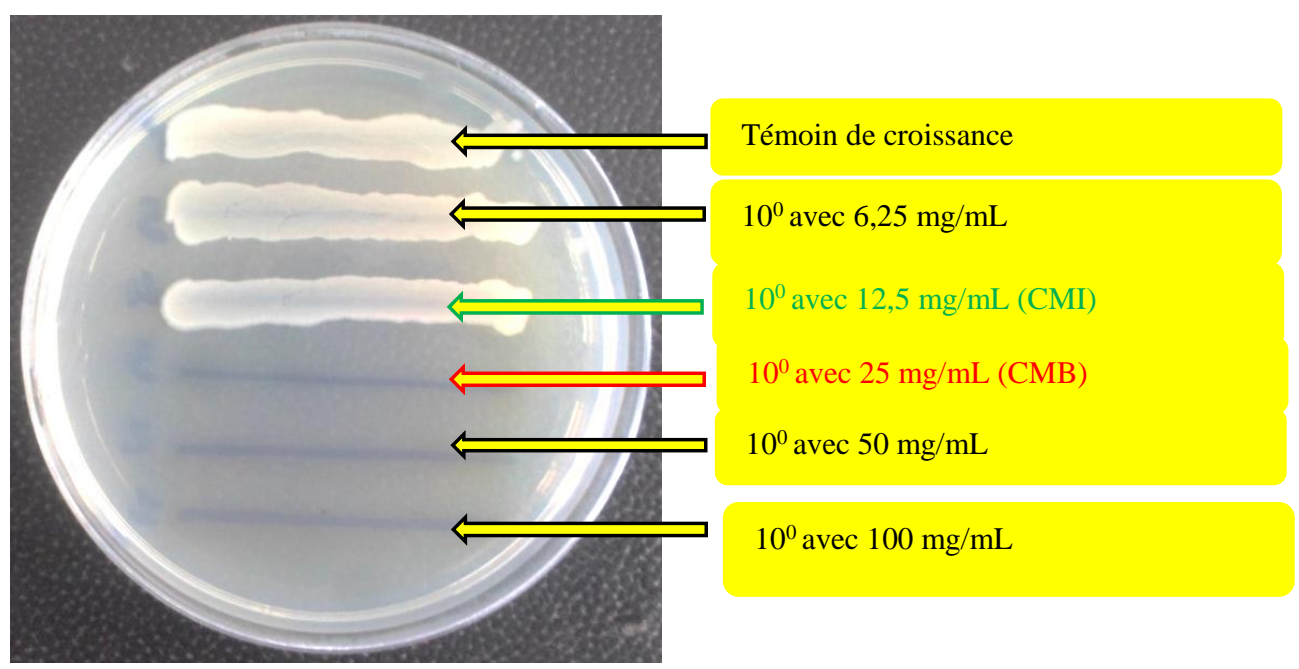

Figure 4: Action de l'extrait hydroéthanolique sur la croissance in vitro des souches de Shigella sp.EEQ.

Tableau 1 : Valeurs des paramètres antibactériens de l'effet des extraits aqueux (E.Taq.) et hydroéthanolique 70\% (E.ETH70\%) sur les souches cliniques de Shigella sp. (EEQ et 1055) testées.

\begin{tabular}{|c|c|c|c|c|c|}
\hline \multirow{2}{*}{ Extrait } & \multirow{2}{*}{ Souches de Shigella sp. } & \multicolumn{2}{|c|}{$\begin{array}{c}\text { Paramètres } \\
\text { antibactériens }(\mathrm{mg} / \mathrm{mL})\end{array}$} & \multirow{2}{*}{$\begin{array}{c}\text { Rapport } \\
\text { CMB/CMI }\end{array}$} & \multirow[b]{2}{*}{ CMB $_{\text {E.Taq }} /$ CMB $_{\text {E.ETH 70\% }}$} \\
\hline & & CMI & CMB & & \\
\hline \multirow{2}{*}{ E.ETH70 } & $\begin{array}{l}\text { Souche clinique } \\
\text { EEQ }\end{array}$ & 12,5 & 25 & 2 & 4 \\
\hline & $\begin{array}{l}\text { Souche clinique } \\
1055\end{array}$ & 6,25 & 25 & 4 & 4 \\
\hline \multirow{2}{*}{ E.Taq. } & $\begin{array}{l}\text { Souche clinique } \\
\text { EEQ }\end{array}$ & 50 & 100 & 2 & 4 \\
\hline & $\begin{array}{l}\text { Souche clinique } \\
1055\end{array}$ & 50 & 200 & 4 & 8 \\
\hline
\end{tabular}

\footnotetext{
E.Taq. : Extrait total aqueux d'Euphorbia hirta.

E.ETH $70 \%$ : Extrait éthanolique $70 \%$ d' Euphorbia hirta.
} 


\section{DISCUSSION}

Le but de cette étude a été d'évaluer l'activité des extraits aqueux et hydroéthanolique d'Euphorbia hirta sur la croissance in vitro de deux souches cliniques de Shigella sp. impliquées dans des diarrhées sanglantes.

L'analyse des rendements montre que l'E.Taq a un rendement (R) plus important, soit $(\mathrm{R}=22,54 \%)$ que l'E.ETH70\% dont $\mathrm{R}=$ $12,21 \%$. Ces valeurs de rendement obtenues sont en accord avec celles indiquées par la Pharmacopée Ouest Africaine.

En effet, celle-ci rapporte que la valeur de substances extractibles à l'eau n'est pas moins de $17,0 \%$ et celle à l'éthanol 70\% n'est pas moins de 11,5\% (OOAS, 2013). Cette différence de rendement pourrait s'expliquer par le fait que le solvant d'extraction éthanol absolu-eau $(70: 30 ; \quad$ V/V $), \quad$ extrait principalement les molécules bioactives et laisse les macromolécules, contrairement à l'eau qui extrait toutes les molécules à cause de sa polarité et sa solubilité (Okou and Yapo, 2018). Cette observation pourrait ainsi expliquer en partie ce rendement élevé de l'extraction à l'eau par rapport à celle à l'éthanol $70 \%$.

La dilution de l'inoculum pur révèle le passage progressif de l'épaisseur de nappes de colonies aux colonies parfaitement isolées au fur et à mesure des dilutions successives décimales, allant de $10^{-1}$ à $10^{-4}$. Cela signifie que les travaux ont été effectués dans les conditions standards de culture, respectant un inoculum de $10^{6} \mathrm{UFC} / \mathrm{mL}$ (Miezan et al., 2017).

L'analyse des résultats de la recherche des activités antibactériennes de l'E.Taq. et de l'E.ETH70\% révèle que les souches cliniques de Shigelles sp. testées sont toutes sensibles à ces extraits d'Euphorbia hirta testés à divers degrés.

En effet, l'observation des paramètres antibactériens montre que les valeurs des CMIs de l'E.ETH70\% varient de 6,25 à $12,5 \mathrm{mg} / \mathrm{mL}$ tandis que celles des CMBs sont de $25 \mathrm{mg} / \mathrm{mL}$ (pour les deux souches 1055, EEQ de Shigella sp., prises dans cet ordre). Il en est de même pour l'E.Taq. dont les CMIs et CMBs prises dans ce même ordre sont respectivement de 50 $\mathrm{mg} / \mathrm{mL}$ (pour les deux souches de Shigella $\mathrm{sp}$ ) et de 100 à $200 \mathrm{mg} / \mathrm{mL}$ respectivement pour la souche Shigella sp EEQ et la souche de Shigella sp 1055. Toutefois, l'E.ETH70\% est quatre à huit fois plus actif que l'E.Taq respectivement pour les inhibitions des souches cliniques de Shigella sp EEQ et 1055 (par référence au rapport d'efficacité sur la base des CMBs soit (CMB E.Taq)/(CMB E.ETH70\%)). Ces résultats montrent que les souches cliniques de Shigella sp. EEQ et Shigella sp 1055 ont pratiquement la même sensibilité à l'E.ETH70\% et plus sensibles à cet extrait qu'à l'E.Taq. Elles ont requis $25 \mathrm{mg} /$ $\mathrm{mL}$ pour leur inhibition, tandis qu'il faut quatre à huit fois plus de cette valeur (soit 100 à 200 $\mathrm{mg} / \mathrm{mL}$ ), pour inhiber les croissances respectives des souches de Shigella sp EEQ et 1055 par l'E.Taq.

Cette meilleure activité anti antishigelle de l'E.ETH70\% par rapport à l'E.Taq. est aussi soutenue par les valeurs plus petites de ses CMIs, telle que l'ont aussi démontré Ouattara et al. (2013). Cela pourrait s'expliquer par le fait que, le solvant hydroéthanolique concentre le mieux les principes actifs antishigelles, contrairement à l'eau qui concentre toutes les molécules (macromolécules et principes actifs) à cause de sa polarité et sa solubilité (Okou and Yapo, 2018).

Les rapports $(\mathrm{CMB} / \mathrm{CMI}=\mathrm{r})$, qui attestent l'efficacité d'une drogue varient de 2 à 4, montrant ainsi que 1'E.ETH70\% et l'E.Taq. ont une activité anti antishigelle bactéricide (Marmonier, 1990 ; Sirot, 1990).

Ces valeurs de CMBs de ces travaux-ci, sont largement au-delà des résultats de travaux de Vijaya et al. (1995), qui ont montré avec l'extrait méthanolique d'Euphorbia hirta, une CMB de $100 \mu \mathrm{g} / \mathrm{mL}$ pour Shigella flexneri et de $200 \mu \mathrm{g} / \mathrm{mL}$ pour Shigella dysenteriae. Cela pourrait s'expliquer par le fait que l'E.ETH70\% moins polaire que le méthanol, 
concentrerait moins les molécules bioactives tandis que ce dernier très polaire serait le solvant qui concentrerait le mieux, les molécules bioactives. Cependant ces résultats actuels selon lesquels l'E.ETH70\% a la meilleure activité antishigella, concordent avec ceux de Gupta et al. (2018). En effet, ceux-ci ont montré que les E.ETH70\% des feuilles, mais aussi des fleurs d'Euphorbia hirta sont plus actifs que les extraits aqueux des feuilles et des fleurs de cette plante sur Escherichia coli, Candida albicans et Staphylococcus aureus.

Le support explicatif de l'activité antibactérienne des extraits d'Euphorbia hirta repose sur l'existence de diverses familles de métabolites secondaires mises en évidence par les tests phytochimiques réalisés par Lanhers (1988) et confirmés par ceux de Soma (2002).

En somme, les actions de l'ETaq. et de l'E.ETH70\% sur l'ensemble des souches cliniques bactériennes étudiées peuvent permettre de dire que ces actions sont dosedépendantes puisqu'elles sont liées à l'augmentation des concentrations des dits extraits. Ces groupes chimiques sont les flavonoïdes, les acides phénoliques, les tanins (catéchique et gallique) et les dérivés terpénique (Lanhers, 1988 ; Soma, 2002).

Les activités antibactériennes des plantes médicinales sont communément dues aux flavonoïdes (N'guessan et al., 2007 ; Ramzi et al., 2010 ; Bagre et al., 2014 ; Bamba et al., 2020), métabolites secondaires dont l'activité antimicrobienne (et donc antiinfectieuse) a été prouvée. En effet, cette activité est due principalement à la capacité de ces molécules d'inhiber l'expression de l'ADN de la synthèse de certaines enzymes et protéines membranaires des microorganismes (Ulanowska et al., 2006 ; Wichtl and Anton, 2009).

\section{Conclusion}

La plante entière d'Euphorbia hirta qui a fait l'objet de cette étude est une plante à laquelle l'on accorde des vertus thérapeutiques contre les diarrhées infectieuses sanglantes en Côte d'Ivoire. Les résultats de ces présents travaux viennent confirmer l'effectivité de l'existence d'agents thérapeutiques, particulièrement des agents antishigelles. L'E.ETH70\%, plus efficace, est quatre à huit fois plus actif que L'E.Taq sur les souches cliniques de Shigelles testées. Les différents extraits de cette plante ont une activité antibactérienne bactéricide sur la croissance in vitro des souches étudiées. Cette action bactéricide observée est dose-dépendante. Les résultats des présents travaux constituent ainsi, une assise scientifique qui vient d'être établie à l'usage traditionnel de cette plante, justifiant ainsi son utilisation dans le traitement des maladies diarrhéiques infectieuses en médecine traditionnelle. Ces travaux contribuent ainsi à la valorisation de la médecine traditionnelle en Côte d'Ivoire. En perspective, il conviendrait d'isoler et d'identifier les molécules responsables de l'activité anti-Shigella.

\section{CONFLITS D'INTERETS}

Les auteurs ne déclarent aucun conflit d'intérêts.

\section{CONTRIBUTIONS DES AUTEURS}

Cette étude a été réalisée en collaboration avec tous les auteurs. Les auteurs KAE, OPA et ADST sont les principaux auteurs de l'article. Tous les autres auteurs ont également contribué à la revue de la littérature et au processus d'édition. Le manuscrit final a été lu et approuvé par les auteurs participants.

\section{REMERCIEMENTS}

Nous remercions le Laboratoire de Bactériologie et de Virologie du Centre Hospitalier Régional (CHR) de Daloa, qui grâce au partenariat existant entre cette structure sanitaire et l'Université Jean Lorougnon GUEDE, nous a fourni les souches pathogènes de Shigelles sur lesquelles nos travaux ont porté. 


\section{REFERENCES}

Adjanohoun EJ, Aké-Assi L. 1979. Contribution au recensement des plantes médicinales en Côte d'Ivoire. Centre National de floristique de l'Université Nationale de Côte d'Ivoire, Tome 1, pp. 23-300.

Aubry P, Gaüzère B. 2019. Diarrhées infectieuses, Actualités 2019. Centre René Labusquière, Institut de Médecine Tropicale, Université de Bordeaux, 33076 Bordeaux (France). 8 p. http://medecinetropicale.free.fr/cours/dia rrhees_infectieuses.pdf

Aubry P, Gaüzère B. 2011. Les maladies liées à l'eau, Actualités 2011, Centre René Labusquière, Institut de Médecine Tropicale, Université de Bordeaux, 33076 Bordeaux (France). 7 p. http://medecinetropicale.free.fr/cours/eau .pdf

Anne-Sophie L. 2018. La Phytothérapie de demain : les plantes médicinales au cœur de la pharmacie. Thèse d'état de pharmacie. Faculté de pharmacie. AixMarseille Université. (France) 92 p. https://dumas.ccsd.cnrs.fr/dumas01840619/document

Bagre I, Ouattara K, Yoro B, Meite S, Coulibaly A. 2014. Mise en évidence des propriétés Antistaphylococciques des flavonoïdes totaux de Thonningia sanguinea (Vahl), une plante de la pharmacopée ivoirienne. Phytothérapie, 12: 360-363. DOI : https://doi.org/10.1007/s10298-0140835-9

Bamba M, Neut C, Bordage S, Dramane S, Kouadio N-J, Yacouba S, Samaillie J, Zamble-Bi TA, Tra-Bi FH, Sahpaz S. 2020. Screening phytochimique des extraits méthanoliques des feuilles de Combretum collinum et des racines de Anogeisus leiocarpus et effet antibactérien in vitro sur des souches de Staphylococcus aureus multirésistantes. Int. J. Biol. Chem. Sci., 14(6): 2362-2372.
DOI:

https://dx.doi.org/10.4314/ijbcs.v14i6.34 Bisht K, Wagner KH, Bulmer AC. 2010. Curcumin, resveratrol and flavonoids as anti-inflammatory, cyto and DNA protective dietary compounds. Toxicology, $\quad \mathbf{2 7 8}(1): \quad 88-100$. DOI: $10.1016 /$ j.tox.2009.11.008

Gupta D, Kumar M, Gupta V. 2018. An in vitro investigation of antimicrobial efficacy of Euphorbia hirta and Murraya koenigii against selected pathogenic microorganisms. Asian $J$ Pharm Clin Res., 11(5): 359-354. DOI: https://doi.org/10.22159/ajpcr.2018 .v11i5.24578

Katarzyna S, Krycińska R, Czepiel J, Garlicki A, Grażyna B. 2019. Diarrhea caused by Shigella flexneri in patients with primary HIV infection. Int. J. STD AIDS, 30(8): 814-816.

DOI: https://doi.org/10.1177/09564624188130 60

Koudoro YA, Bogninou GSR, Bossou AFAD, Agbangnan DCP, Olayé T, Bothon FTD, Alitonou GA, Avlessi F, Sohounhloue D. 2019. Métabolites secondaires, activités antibactérienne et antiradicalaire des extraits de l'écorce de tronc de Acacia polyacantha récoltée au Benin. Int. J. $A d v$. Res., 7(10) : 1087-1092. DOI: 10.21474/IJAR01/9927

Lanhers MC. 1988. Contribution à l'étude ethnobotanique et pharmacologique d'Euphorbia hirta L.: Propriétés Psychotropes, Analgésiques, Antiinflammatoires. Thèse de Doctorat, Université de Lauraine, Metz (France), $692 \mathrm{p}$.

Lehmann H. 2014. Le médicament à base de plantes en Europe : statut, enregistrement, contrôles. Thèse de doctorat. Faculté de Pharmacie. Université de Strasbourg, (France), 342 p. https://tel.archivesouvertes.fr/tel-00936734/document

Marmonier AA. 1990. Technique de diffusion en gélose: Méthode des disques. 
In Bactériologie Médicale, Techniques

Usuelles. Doin : Paris, France ; 227-244.

Miezan BAP, Koffi AE, By P, Okpekon AT,

Yapi HF. 2017. Study of the in vitro antibacterial activity of the aqueous and hydroethanolic extracts from the leaves of the Erythrococca anomala (Euphorbiaceae) on six bacterial strains. Int J. Biochem. Biophys., 5(1): 19-25. DOI: 10.13189/ijbb.2017.050103

Logan NZ, Karp BE, Tagg KA, BurnsLynch C, Chen J, Garcia-Williams A, Marsh ZA, O'Laughlin K, Plumb ID, Schroeder MN, Webb HE, Zenas H, Draper J, Ginn A, Martinez E, Partridge SR, Sim E, Sintchenko V, Iredell J, Watkins LF. 2020. Increase in Multidrug Resistance (2011-2018) and the Emergence of Extensive Drug Resistance (2020) in Shigella sonnei in the United States. Open Forum Infect. Dis., 7(1): S195.

DOI: https://doi.org/10.1093/ofid/ofaa439.440

N'guessan JD, Zirihi GN, Kra AKM, Kouakou K, Djaman AJ, Guédé-Guina F. 2007. Free radical scavenging activity, flavonoid and phenolic contents of selected Ivoirian plants. IJONAS, 4: 425429. DOI: 10.4314/ijonas.v3i4.36155

Okou OC, Yapo ES. 2018. Activité antibactérienne des extraits de feuilles de solanum torvum: Extraits de feuilles de Solanum torvum (Solanaceae) sur la croissance in vitro de 3 souches d'entérobactéries. Edition Universitaires Européennes, Berlin (Allemagne), $40 \mathrm{p}$.

OOAS (Organisation Ouest Africaine de la Santé). 2013. Pharmacopée Ouest Africaine: Euphorbia hirta. Organisation Ouest Africaine de la Santé ; 81-85.

OMS (Organisation Mondiale de la Santé) 2004. Le point sur les épidémies. $R E H$. 79(39): 349-356. http://www.who.int/wer/2004/en/wer793 9.pdf

Ouattara A, Traore Y, Ouattara GA, Konate G, Ouattara K, Coulibaly A. 2020.
Antioxidant and anti-gastroenteritis activities of Funtumia elastica (Apocynaceae) and Caesalpinia bonduc (Caeasalpiniaceae). Int. J. Biol. Chem. Sci., 14(1): 170-180. DOI: https://dx.doi.org/10.4314/ijbcs.v14i1.14

Ouattara K, Doumbia I, Touré A, Djaman AJ, Coulibaly A. 2013. Activité antibactérienne des extraits des feuilles de Morinda morindoides (Morinda, Rubiaceae) sur Staphylococcus aureus et Pseudomonas aeruginosa. Phytothérapie, 11: 172-177. DOI 10.1007/s10298-013-0780-z

Tai AYC, Easton M, Encena J, Rotty J, Valcanis M, Howden BP, Slota-Kan S, Gregory J. 2016. A review of the public health management of shigellosis in Australia in the era of cultureindependent diagnostic testing. Aust $N \mathrm{Z}$ $J$ Public Health, 40(6): 588-591. DOI: 10.1111/1753-6405.12590

Ramzi AAM, Salah AAA, Sidgi H, Faisal MNA, Sama AZA, Urlike L. 2010. Antimicrobial, antioxidant and cytotoxic activities and phytochemical screening of some Yemeni medicinal plants. Evid Based Complement Alternat Med., 7: 323-330. DOI: 10.1093/ecam/nen004

Robert C, Edouard B, Dominique G, Olivier P, Francine Gr-CNR, Françoise D, Véronique V (InVS), Dominique DejourSalamanca, Ségovia-Kueny S, Nathalie $\mathrm{D}$, Charlier-Bret N, Isabelle Pe, Agence française de sécurité sanitaire des produits de santé (Afssaps). 2004. Traitement antibiotique des gastroentérites à Shigella sonnei. Presse Med., 33(21): $\quad$ 1538-1545. DOI : https://doi.org/10.1016/S07554982(04)98980-4

Sekandi P, Namukobe J, Byamukama R, Akala HM, Yeda RA, Heydenreich M. 2020. Bioactive secondary metabolites from the leaves of Secamone africana (Olive.) Bullock. Int. J. Biol. Chem. Sci., 14(5): 
1820-1830.

DOI:

https://doi.org/10.4314/ijbcs.v14i5.25

Sirot J. 1990. Evaluation de l'activité antibactérienne des antibiotiques in vitro. In Bactériologie Médicale (2 ${ }^{\mathrm{ème}}$ édition). Flammarion ; 297-315.

Soma OB. 2002. Activité antibactérienne d'extraits d'Euphorbia hirta (Linn), une plante utilisée traditionnellement dans le traitement des infections urinaires. Thèse de Doctorat, UFR des Sciences de la Santé Section Pharmacie, Université de Ouagadougou (Burkina-Faso), $110 \mathrm{p}$.

Ulanowska K, Traczyk A, Konopa G, Wegrzym G. 2006. Differential antibacterial activity of genistein arising from global inhibition of DNA, RNA and protein synthesis in some bacterial strains. Arch Microbiol., 184(5): 271278. DOI: $10.1007 / \mathrm{s} 00203-005-0063-7$

Vijaya K, Ananthan S, Nalini R. 1995. Antibacterial effect of theaflavin, polyphenon 60 (Camellia sinensis) and Euphorbia hirta on Shigella spp, a cell culture study. J. Ethnopharmacol., 49(2): 115-118. DOI: $\quad 10.1016 / 0378-$ 8741(95)90039-x.

Wichtl M, Anton R. 2009. Plantes thérapeutiques. Tradition, pratique officinale, science et thérapeutique. Lavoisier : $\quad$ Paris ; 38-41. https://www.unitheque.com/plantestherapeutiques/tec-doc-inter/Livre/3248

Yolidje I, Keita AD, Moussa I, Toumane A, Bakasso S, Saley K, Much T, Pirat JL, Ouamba JM. 2020. Enquête ethnobotanique sur les plantes utilisées traditionnellement au Niger dans la lutte contre les moustiques vecteurs des maladies parasitaires. Int. J. Biol. Chem. Sci., 14(2): 570-579. DOI: https://dx.doi.org/10.4314/ijbcs.v14i2.21 